\section{TUBERCULOSE PULMONAR ENTRE USUÁRIOS DE UMA UNIDADE DE ATENÇÃO BÁSICA}

\author{
Pulmonary tuberculosis among users of a primary healthcare \\ unit
}

\author{
Tuberculosis pulmonar en los usuarios de una unidad de \\ atención básica
}

\section{RESUMO}

Objetivo: Avaliar a prevalência de casos bacilíferos de tuberculose pulmonar entre usuários de uma unidade de atenção básica. Métodos: Estudo observacional, de corte transversal, realizado entre fevereiro e agosto de 2011 com 1.873 indivíduos maiores de 18 anos atendidos na Unidade Básica de Saúde (UBS), localizada na zona norte do município do Rio de JaneiroRJ (Brasil), selecionados por conveniência de forma não probabilística. Inicialmente, aplicouse instrumento para o diagnóstico presuntivo de tuberculose pulmonar. Os indivíduos com escore positivo foram submetidos ao teste bacilífero. Realizou-se estatística descritiva com frequência simples e percentual, bem como teste exato de Fisher para avaliar a associação com sexo, idade e os resultados da baciloscopia de escarro. Resultados: Entre os indivíduos atendidos na UBS estudada, $157(8,4 \%)$ apresentaram escore positivo para diagnóstico presuntivo de tuberculose. Nesses, os sintomas mais frequentes foram tosse $(76,5 \%, n=13)$ e dor torácica $(70,6 \%, n=12)$. Não houve associação significativa entre baciloscopia positiva com sexo $(p=0,477)$ e idade $(p=0,186)$. Conclusão: A ocorrência de casos bacilíferos de tuberculose pulmonar entre indivíduo com escore positivo de presunção dessa doença mostrou-se alta na unidade de saúde estudada.

Descritores: Tuberculose Pulmonar; Diagnóstico; Programas de Rastreamento; Atenção Primária à Saúde.

\section{ABSTRACT}

Objective: To evaluate the prevalence of positive bacterial sputum cases of pulmonary tuberculosis among users of a primary healthcare unit. Methods: Cross-sectional, observational study, conducted between February and August 2011 with 1,873 individuals aged above 18 years seen at a Basic Healthcare Unit (BHU) in the city of Rio de Janeiro, Brazil, selected through convenience non-probabilistic sampling. A tool for the presumptive diagnosis of pulmonary tuberculosis was initially applied. The individuals with positive score were submitted to bacilloscopy. Descriptive statistics was performed, with absolute and percentage frequencies, as well as Fisher's exact test to assess the association between gender, age, and the results of bacterial sputum microscopy. Results: Of the users seen at the studied BHU, 157 individuals were tested positive for the presumptive diagnosis of tuberculosis. Their most frequent symptoms were cough $(76.5 \%, n=13)$ and chest pain $(70.6 \%, n=12)$. There was no significant association between positive bacilloscopy, sex $(p=0.477)$, and age $(p=0.186)$. Conclusion: The frequency of positive bacilloscopy cases of pulmonary tuberculosis among individuals with positive score for presumption of that disease was high in the studied health unit.

Descriptors: Tuberculosis, Pulmonary; Diagnosis; Mass Screening; Primary Health Care.
Artigo Original
1) Universidade Iguaçu - UNIG - Nova Iguaçu - (RJ) - Brasil.

2) Faculdade de Enfermagem, Universidade do Estado do Rio de Janeiro - UERJ - Rio de Janeiro (RJ) - Brasil.

3) Escola de Enfermagem Anna Nery, Universidade Federal do Rio de Janeiro -

UFRJ - Rio de Janeiro (RJ) - Brasil.

Recebido em: 28/08/2014 Revisado em: $15 / 11 / 2014$ Aceito em: 14/12/2014 


\section{RESUMEN}

Objetivo: Evaluar la prevalencia de los casos baciliferos de tuberculosis pulmonar de los usuarios de una unidad de atención básica. Métodos: Estudio observacional de corte transversal realizado entre febrero y agosto de 2011 con 1873 individuos mayores de 18 años asistidos en la Unidad Básica de Salud (UBS) localizada en la zona norte del municipio de Rio de Janero-RJ (Brasil), elegidos por conveniencia y muestreo no probabilístico. En principio se aplicó un instrumento para el diagnóstico presuntivo de tuberculosis pulmonar. Los individuos con la puntuación positiva fueron sometidos al test bacilifero. Se realizó la estadística descriptiva con frecuencia simple y porcentual así como el test exacto de Fisher para evaluar la asociación del sexo, la edad y los resultados de la baciloscopia de esputo. Resultados: De los individuos asistidos en la UBS investigada, 157 (8,4\%) presentaron puntuación positiva para el diagnóstico presuntivo de tuberculosis. En ellos, los síntomas más frecuentes fueron la tos $(76,5 \%, n=13)$ y el dolor torácico $(70,6 \%, n=12)$. No hubo asociación significativa de la baciloscopia positiva y el sexo $(p=0,477)$ y la edad $(p=0,186)$. Conclusión: La presencia de los casos baciliferos de tuberculosis pulmonar en individuos con puntuación positiva de presunción para esta enfermedad fue elevada en la unidad de salud investigada.

Descriptores: Tuberculosis Pulmonar; Diagnóstico; Tamizaje masivo; Atenção Primaria de Salud.

\section{INTRODUÇÃO}

A tuberculose pulmonar (TB) é um importante problema de saúde no mundo que exige o desenvolvimento de estratégias para o seu controle, considerando aspectos humanitários, econômicos e de saúde pública. No contexto mundial, o Brasil é um dos 22 países que, juntos, foram responsáveis por $80 \%$ de todos os casos de TB no ano de 2012, sendo priorizados pela Organização Mundial de Saúde (OMS) para reduzir à metade essa incidência e mortalidade ${ }^{(1)}$.

Em 2012, foram notificados 70.047 casos novos de TB no Brasil, o que correspondeu a um coeficiente de incidência de 36,1/100.000 habitantes. O Rio de Janeiro foi o estado brasileiro com o maior coeficiente de incidência de TB (68,7/100.000 habitantes), tendo registrado 14.039 casos novos, representando cerca de $20 \%$ do total do país. Mais da metade desses casos $(52,94 \%)$ ocorreram na capital, Rio de Janeiro, que apresentou um coeficiente de incidência de 91,2/100.000 habitantes ${ }^{(2)}$.

Diante dessas constatações, destaca-se que, para interromper a cadeia de transmissão de TB na comunidade, a principal estratégia de detecção precoce de doentes bacilíferos para TB deve ser baseada na captação ativa de sintomáticos respiratórios, na realização de baciloscopia de escarro e no pronto tratamento dos casos $\operatorname{positivos}^{(3)}$. Sintomático respiratório é o indivíduo que apresenta tosse produtiva (que expele muco) por, pelo menos, três semanas $^{(1)}$, e que deve ser captado ativamente, através de rastreamentos, na população com 15 ou mais anos de idade $^{(4,5)}$.

Portanto, a captação ativa de sintomáticos respiratórios deve ser a estratégia priorizada e realizada permanentemente pelos serviços de saúde de todos os níveis de atenção para a descoberta de casos bacilíferos de $\mathrm{TB}^{(1,5,6)}$. Todavia, é um grande desafio identificar precocemente sintomáticos respiratórios e indivíduos com maior potencial de diagnóstico de TB entre os usuários da atenção básica. A identificação do sintomático respiratório somente quando este chega à Unidade Básica de Saúde (UBS) para procura espontânea do diagnóstico pode não levar à descoberta de todos os casos existentes na população, pois a tosse, quando prolongada, pode não ser percebida como um sintoma que leve o indivíduo a buscar a UBS ${ }^{(6,7)}$.

O objetivo deste estudo foi identificar casos bacilíferos de TB entre usuários de uma unidade de atenção básica do município do Rio de Janeiro.

\section{MÉTODOS}

Estudo observacional, de corte transversal, desenvolvido em uma UBS localizada na zona norte do município do Rio de Janeiro-RJ (Brasil), em 2011.

Durante o período de realização da pesquisa, a UBS em questão encontrava-se em um processo de transição para atender completamente aos moldes da Estratégia Saúde da Família, mas três equipes mínimas de Saúde da Família (um médico, um enfermeiro, um técnico de enfermagem e seus agentes comunitários de saúde - por equipe) já eram responsáveis pelos cuidados básicos de saúde de 10.428 pessoas. Além disso, a UBS continuava a atender à demanda espontânea e agendada de usuários residentes em um dos 28 bairros adjacentes a ela, que, em média, possuíam um índice de desenvolvimento humano (IDH) de $0,6^{(8)}$.

Três entrevistadores devidamente treinados para a coleta de dados abordaram 1.873 indivíduos de ambos os sexos, com idade maior que 18 anos, que compareceram à UBS por qualquer motivo de atendimento, entre fevereiro e agosto de 2011. Foram excluídos do estudo as gestantes e os indivíduos já diagnosticados e/ou em tratamento para TB.

A abordagem dos indivíduos não foi probabilística e por conveniência, e acontecia duas vezes por semana (de segunda a sexta-feira), entre 8 e 17 horas (horário de funcionamento da UBS). Na sala de espera da unidade de saúde, um entrevistador abordava o indivíduo, informando-o sobre a natureza e os objetivos da pesquisa. Sua participação era voluntária e era lido o Termo de Consentimento Livre e 
Esclarecido. Em seguida, aqueles que aceitavam participar eram entrevistados em local reservado e sem a presença de terceiros. As entrevistas duraram aproximadamente 10 minutos.

Inicialmente, os indivíduos incluídos no estudo responderam a um escore clínico de alta sensibilidade para o diagnóstico presuntivo de $\mathrm{TB}^{(9)}$. Este instrumento é composto por questões de fácil resposta, sem causar constrangimento na entrevista de triagem; sua pontuação varia de zero a 22, o que gera um sistema de escore para a aplicação em pacientes com suspeita de TB. Na validação desse escore clínico, o ponto de corte $\geq 8$ apresentou melhor relação entre a sensibilidade $(83,1 \%)$ e a acurácia $(58,6 \%)$. A área sob a curva ROC para o escore em relação à TB foi de $0,734^{(9)}$.

Dentre os 1.873 indivíduos abordados, 176 apresentaram escore positivo de presunção de TB (pontuação $\geq 8$ ) e foram convidados a participar deste estudo. Houve 19 recusas. A amostra foi composta por 157 indivíduos.

Os indivíduos que compuseram a amostra responderam a um questionário que contemplava as seguintes variáveis: sexo (masculino e feminino); faixa etária (18-36, 37-58, $\geq 59$ anos); situação conjugal (casado, separado, solteiro e viúvo); escolaridade (analfabeto, ensino fundamental incompleto, ensino fundamental completo, ensino médio completo e ensino superior completo); renda familiar mensal (até 1 salário mínimo, 1 salário mínimo, 2-3 salários mínimos, 4-5 salários mínimos e $\geq 6$ salários mínimos).

Os indivíduos foram submetidos à baciloscopia de escarro em duas amostras que, quando positivas, foram utilizadas como critério diagnóstico de TB pulmonar ${ }^{(5)}$.

Os dados coletados foram digitados e analisados no programa Epi Info, versão 3.5.1, por meio de distribuição de frequências simples. O teste exato de Fisher foi empregado nas análises adotando-se um nível de significância de 0,05 para verificar a associação entre variáveis sexo e idade e os resultados da baciloscopia de escarro.

O presente estudo foi aprovado pelo Comitê de Ética em Pesquisa da Secretaria Municipal de Saúde e Defesa Civil do Rio de Janeiro, sob número de protocolo 135A/2011, e seguiu a Resolução 466/12 do Conselho Nacional de Saúde.

A UBS em que este estudo foi desenvolvido tinha um Programa de Controle da Tuberculose, para o qual foram encaminhados os casos de TB diagnosticados durante a pesquisa. Os indivíduos que apresentaram escores positivos de presunção de TB e se recusaram a participar do estudo foram informados sobre a importância de investigar adequadamente os sinais e sintomas apresentados, sendo encorajados a buscar o Programa de Controle da Tuberculose da unidade de saúde para a realização de exames complementares (baciloscopia de escarro e radiografia de tórax).

\section{RESULTADOS}

A população deste estudo foi constituída por $9,4 \%$ $(n=176)$ de indivíduos com escore clínico presuntivo de $\mathrm{TB}$, dos quais 19 se recusaram a realizar o teste bacilífero. Dos 157 que continuaram no estudo, a maioria era do sexo masculino $(56,1 \%, \mathrm{n}=88)$, solteira $(53,5 \%, \mathrm{n}=84)$, com faixa etária entre 37-58 anos $(52,2 \%, n=82)$, média de 42,8 anos $( \pm 15,9)$. Informaram possuir o ensino médio completo $52,2 \%(n=82)$ dos entrevistados, além de 41,4\% $(n=65)$ terem renda familiar mensal de um salário mínimo.

Após realização da bacilocospia, 10,8\% $\quad(n=17)$ apresentaram escarro positivo, sendo considerados, portanto, casos bacilíferos de TB (Tabela I).

Tabela I - Características sociodemográficas e resultados da baciloscopia de escarro dos usuários com suspeição de tuberculose da amostra. Rio de Janeiro-RJ, 2011. (n=157)

\begin{tabular}{|c|c|c|}
\hline Variáveis & $\mathbf{n}$ & $\%$ \\
\hline $\begin{array}{l}\text { Sexo } \\
\text { Feminino }\end{array}$ & 69 & 43,9 \\
\hline Masculino & 88 & 56,1 \\
\hline $\begin{array}{l}\text { Faixa etária } \\
18-36\end{array}$ & 56 & 35,7 \\
\hline $37-58$ & 82 & 52,2 \\
\hline $\begin{array}{l}\geq 59 \\
\text { Situacão conjugal }\end{array}$ & 19 & 12,1 \\
\hline $\begin{array}{l}\text { Casado } \\
\text { Separado } \\
\text { Solteiro } \\
\text { Viúvo }\end{array}$ & $\begin{array}{l}59 \\
12 \\
84 \\
2\end{array}$ & $\begin{array}{r}37,6 \\
7,6 \\
53,5 \\
1,3\end{array}$ \\
\hline Escolaridade & & \\
\hline Analfabeto & 6 & 3,8 \\
\hline Fundamental incompleto & 17 & 10,8 \\
\hline Fundamental completo & 48 & 30,6 \\
\hline Ensino médio completo & 82 & 52,2 \\
\hline $\begin{array}{l}\text { Ensino superior completo } \\
\text { Renda familiar mensal }\end{array}$ & 4 & 2,6 \\
\hline Até 1 salário mínimo & 25 & 15,9 \\
\hline 1 salário mínimo & 65 & 41,4 \\
\hline 2-3 salários mínimos & 53 & 33,7 \\
\hline 4-5 salários mínimos & 10 & 6,4 \\
\hline $\begin{array}{l}\geq 6 \text { salários mínimos } \\
\text { Baciloscopia de escarro }\end{array}$ & 4 & 2,6 \\
\hline Negativa & 140 & 89,2 \\
\hline Positiva & 17 & 10,8 \\
\hline
\end{tabular}

Segundo o escore clínico para o diagnóstico presuntivo de TB, os sintomas mais frequentes entre os casos bacilíferos identificados pelo estudo foram tosse $(76,5 \%, n=13)$ e dor torácica $(70,6 \%, \mathrm{n}=12)$ (Tabela II). 
Tabela II - Sintomas apresentados pelos casos bacilíferos de tuberculose identificados pelo estudo. Rio de Janeiro-RJ, 2011. $(\mathrm{n}=17)$

\begin{tabular}{lcc}
\hline Sintomas & $\mathbf{n}$ & $\mathbf{\%}$ \\
\hline Dor torácica & 12 & 70,6 \\
Sim & 5 & 29,4 \\
Não & & \\
Tosse & 13 & 76,5 \\
Sim & 4 & 23,5 \\
Não & & \\
Expectoração & 9 & 52,9 \\
Sim & 8 & 47,1 \\
Não & & \\
Hemoptise & 4 & 23,5 \\
Sim & 13 & 76,5 \\
Não & & \\
Sudorese noturna & 6 & 25,3 \\
Sim & 11 & 64,7 \\
Não & & \\
Febre & 6 & 25,3 \\
Sim & 11 & 64,7 \\
Não & & \\
Perda de peso & 7 & 41,2 \\
Sim & 10 & 58,8 \\
Não & & \\
\hline
\end{tabular}

Tabela III - Associação entre os resultados da baciloscopia de escarro e variáveis selecionadas. Rio de Janeiro-RJ, 2011. $(\mathrm{n}=157)$

\begin{tabular}{lccc}
\hline & \multicolumn{2}{c}{ Baciloscopia de escarro } & \\
\cline { 2 - 3 } Variáveis & $\begin{array}{c}\text { Positiva } \\
\mathbf{n}(\%)\end{array}$ & $\begin{array}{c}\text { Negativa } \\
\mathbf{n}(\%)\end{array}$ & p valor* \\
\hline Sexo & & & \\
Feminino & $9(10,2)$ & $79(89,8)$ & \\
Masculino & $8(11,5)$ & $61(88,4)$ & 0,477 \\
Total & $17(10,8)$ & $140(89,2)$ & \\
Faixa etária & & & \\
18-35 anos & $09(16,4)$ & $46(83,6)$ & \\
$36-58$ anos & $08(9,8)$ & $74(90,2)$ & 0,186 \\
$\geq 59$ anos & - & $20(100)$ & \\
Total & $17(10,8)$ & $140(89,2)$ & \\
\hline
\end{tabular}

* Teste exato de Fisher

Não foram observadas associações estatisticamente significativas entre os resultados da baciloscopia de escarro e as variáveis sexo $(\mathrm{p}=0,477)$ e faixa etária $(\mathrm{p}=0,186)$ (Tabela III).

\section{DISCUSSÃO}

No presente estudo, a proporção de indivíduos com diagnóstico presuntivo de TB identificados como casos bacilíferos de TB pulmonar foi considerada elevada
(10,8\%), porém, não foi encontrada associação com sexo e faixa etária, sendo os principais sintomas relatados tosse e dor torácica.

Desse modo, reforça-se a necessidade do rastreamento de casos de TB pulmonar, com o objetivo de interromper a cadeia de transmissão através do diagnóstico precoce e tratamento imediato da doença em unidades de atenção básica, seja através da identificação de pessoas com sintomas altamente sugestivos de TB e/ou de sintomáticos respiratórios. Medidas de deteç̧ão precoce de doentes bacilíferos para TB mais rápidas e eficazes devem ser instituídas sistematicamente nos serviços de atenção básica, sobretudo em municípios com alta incidência de TB.

Estudos realizados para conhecer a proporção de sintomáticos respiratórios em níveis locais de assistência à saúde da população são importantes, visto que servem de parâmetros para o planejamento de ações operacionais do Programa de Controle de Tuberculose nos municípios em que são desenvolvidos ${ }^{(7)}$.

O Programa Nacional de Controle da Tuberculose recomenda, para fins operacionais, que se considere uma prevalência de $5 \%$ de sintomáticos respiratórios nas consultas de primeira vez dos indivíduos maiores de 15 anos nas unidades de saúde ou de $1 \%$ da população a ela adscrita $^{(5)}$. Com base nos indivíduos captados em serviços brasileiros de atenção básica, a prevalência de sintomáticos respiratórios varia de 4,0 a $10,7 \% \%^{(4,7,10-12)}$.

No México, foram encontrados 3,6\% de sintomáticos respiratórios entre os usuários da atenção primária ${ }^{(13)}$. Nos serviços de atenção primária à saúde da Índia, foram encontrados 2,5\% de sintomáticos respiratórios, com incremento para $4,2 \%$ quando se considerou o tempo de tosse a partir de duas semanas ${ }^{(14)}$, parâmetro esse já levado em consideração no estudo em questão. Resultados semelhantes com os demais estudos brasileiros foram encontrados no presente estudo, em que 9,3\% ( $\mathrm{n}=176)$ dos entrevistados apresentaram sintomas de TB, segundo o escore clínico utilizado, muito acima do encontrado no México e na Índia.

No Rio de Janeiro, estudando-se a prevalência de casos de TB entre sintomáticos respiratórios que buscavam atendimento em unidade de saúde, foram encontrados 10,7\% de usuários com tosse com duração de mais de uma semana, e entre eles, uma prevalência de TB pulmonar de $2,7 \%{ }^{(12)}$.

Apesar de serem poucos os estudos realizados no Brasil sobre a prevalência de sintomáticos respiratórios captados na comunidade, os resultados do estudo citado não são diferentes dos encontrados naqueles realizados em unidades de saúde.

Não foi observada no estudo em questão uma associação estatisticamente significativa entre os resultados da baciloscopia de escarro e as variáveis sexo e faixa 
etária. No entanto, a associação entre TB ativa e o sexo masculino já foi descrita anteriormente ${ }^{(15)}$. É possível que essa associação se deva ao fato de que os homens são mais propensos à exposição a certos fatores de risco para tubérculo, como consumo de bebidas alcoólicas, uso de drogas ilícitas, ser ex-presidiário e fumante ${ }^{(16)}$. A associação entre faixa etária $(<40$ anos $)$ e TB pulmonar também foi encontrada em outros estudos ${ }^{(12,17)}$.

Semelhante aos resultados de outras investigações ${ }^{(18,19)}$, a presença de tosse neste estudo foi referida por $76,5 \%$ dos sujeitos identificados com TB. Conforme recomendações da $\mathrm{OMS}^{(1)}$, do Ministério da Saúde ${ }^{(5)}$ e da literatura cientifica ${ }^{(6,7)}$, a procura de casos novos de TB deve ser realizada por meio da busca de pessoas que apresentem tosse por mais de três semanas, justificando a importância de estudar esse sintoma. Todavia, a tosse com pelo menos uma semana de duração como um sintoma presuntivo de TB deve ser valorizada em usuários de UBS localizadas em áreas de alta prevalência dessa doença, conforme já descrito pela literatura ${ }^{(12)}$.

A fim de que ocorram ações de controle da TB pulmonar na atenção básica, é fundamental a conscientização, o envolvimento, a integração e a articulação permanente dos responsáveis pelo controle dessa doença nos diversos níveis do sistema de saúde, para a viabilização de políticas, planejamento, avaliação e adequação em conjunto com as estratégias e tecnologias adotadas ${ }^{(20)}$.

Destaca-se, no entanto, que há diversos elementos que concorrem para que a atenção à TB dispensada na atenção básica não contemple sua resolutividade, corroborando para que essa doença ainda permaneça como problema de saúde pública. Um dos pontos cruciais é a forma de organização dos serviços de saúde em âmbito local, que tem privilegiado a lógica da atenção à demanda espontânea em detrimento da necessidade de reorganização dos serviços sob o prisma das condições crônicas que exigem resolutividade na atenção. Deve ser efetivada a qualificação dos profissionais para lidar com doenças que envolvem aspectos culturais, sociais, econômicos e de subjetividades, tal qual a $\mathrm{TB}^{(21)}$.

Portanto, é importante que sejam realizadas ações em saúde voltadas para a orientação contínua e esclarecedora sobre a TB e suas formas de transmissão, sintomas e diagnóstico precoce, dirigidas aos usuários de serviços básicos de saúde, a fim de que o envolvimento entre os profissionais e a comunidade, proveniente dessas ações, possa contribuir para o controle da TB no Brasil ${ }^{(22)}$.

Um estudo que objetivou analisar o envolvimento de equipes de atenção básica nas ações de controle da TB na percepção dos coordenadores do Programa de Controle da Tuberculose de nove municípios de São Paulo apontou o descompasso entre a transferência de responsabilidades das ações de controle da TB para a atenção básica e a inadequação quantitativa e qualitativa de recursos humanos para o manejo da doença no sistema de saúde, ressaltando que a incorporação da busca de sintomáticos respiratórios e tratamento supervisionado na atenção básica é necessária para a elaboração de uma política de recursos humanos que garanta a formação e a capacitação contínua de seus profissionais ${ }^{(20)}$.

$\mathrm{Na}$ atenção básica, a busca ativa de sintomáticos respiratórios para diagnóstico precoce de casos de TB é tarefa do agente comunitário de saúde (ACS). A fim de avaliar essa atividade, um estudo analisou a percepção dos enfermeiros supervisores do Programa Agentes Comunitários de Saúde (PACS) de um distrito de saúde do município de Ribeirão Preto-SP e apontou debilidades referentes ao processo de conformação e organização do PACS; dentre elas, destacouse a capacitação insuficiente dos $\operatorname{ACS}^{(23)}$.

Um estudo que analisou o conhecimento de ACS no controle da tuberculose e a autopercepção do seu nível de conhecimento e de sua importância no enfrentamento da doença no município de Vitória-ES obteve resultados semelhantes aos do estudo anteriormente citado $^{(24)}$. De maneira geral, os ACS possuíam conhecimento muito limitado da sintomatologia clássica da tuberculose, em especial no que se refere à tosse prolongada e, por conseguinte, quanto à definição de um sintomático respiratório.

Outra pesquisa, também realizada no município de Vitória-ES, identificou que enfermeiros e médicos possuíam conhecimento suficiente sobre a tuberculose. No entanto, os resultados dessa pesquisa apontaram que não era o conhecimento, mas outros fatores que influenciavam a manutenção de índices elevados da doença no município (como a deficiente identificação precoce de sintomáticos respiratórios que buscam espontaneamente a unidade de saúde), apontando para diferenças existentes entre as categorias profissionais que parecem indicar a pouca socialização do conhecimento na equipe multidisciplinar ${ }^{(25)}$.

Ainda que o paciente, ao chegar ao serviço de saúde, seja atendido em sua demanda, uma das principais dificuldades ainda é a identificação precoce desse mesmo paciente como um sintomático respiratório no acesso ao serviço de saúde e também na comunidade. Logo, a implantação efetiva da estratégia de busca ativa de casos de TB perpassa pelo investimento no treinamento dos ACS, que possuem um papel fundamental no controle da tuberculose ${ }^{(24)}$. Isso pode ser observado no estudo em questão, no qual a amostra foi retirada de pessoas que estavam na UBS em busca de atendimento, mas não necessariamente por causa de seus sintomas respiratórios

Em suma, infere-se que, durante a realização de consultas médicas e de enfermagem, bem como durante 
as visitas domiciliares realizadas pelos ACS, a utilização de instrumentos de rastreamento que possam acelerar a investigação laboratorial e aumentar a detecção da TB pulmonar pode contribuir para o início precoce do tratamento e tornar-se uma ferramenta útil em unidades de atenção básica. Destaca-se, portanto, o desempenho do escore clínico presuntivo de $\mathrm{TB}^{(9)}$ utilizado neste estudo, que se mostrou de fácil utilização pela equipe de entrevistadores.

Convém mencionar que este estudo apresenta limitações. Por ter sido realizado em apenas uma UBS, seus achados e conclusões podem não ser aplicáveis a outras unidades do município do Rio de Janeiro; outra limitação é o critério diagnóstico de TB utilizado neste estudo (baciloscopia de escarro), pois, embora tal critério tenha elevado o valor preditivo positivo em nosso meio, o diagnóstico de certeza de TB somente é feito com a cultura positiva para $M$. tuberculosis. A recusa na participação de $10,8 \%$ dos indivíduos com diagnóstico presuntivo de TB pode ter refletido em uma frequência menor de casos bacilíferos de TB encontrada por este estudo, além de a amostra ser pequena e do tipo não probabilística.

\section{CONCLUSÃO}

A ocorrência de casos bacilíferos de tuberculose pulmonar entre indivíduos com escore positivo de presunção dessa doença mostrou-se alta na unidade de saúde estudada.

\section{REFERÊNCIAS}

1. World Health Organization - WHO. Global tuberculosis report 2013. Geneva: WHO; 2013.

2. Ministério da Saúde (BR). Banco de dados do Sistema Único de Saúde - DATASUS [acesso em 2013 Set 10]. Disponível em: http://www.datasus.gov.br

3. World Health Organization - WHO. Treatment of tuberculosis: guidelines. Geneva: WHO; 2010.

4. Freitas FTM, Yokota RTC, Castro APB, Andrade SSCA, Nascimento GL, et al. Prevalência de sintomáticos respiratórios em regiões do Distrito Federal, Brasil. Rev Panam Salud Pública. 2011;29(6):451-6.

5. Ministério da Saúde (BR). Manual de recomendações para o controle da tuberculose no Brasil. Brasília: Ministério da Saúde; 2011.

6. Golub JE, Mohan CI, Comstock GW, Chaisson RE. Active case finding of tuberculosis: historical perspective and future prospects. Int $\mathrm{J}$ Tuberc Lung Dis. 2005;9(11):1183-203.
7. Moreira CMM, Zandonade E, Lacerda T, Maciel ELN. Sintomáticos respiratórios nas unidades de atenção primária no Município de Vitória, Espírito Santo, Brasil. Cad Saúde Pública. 2010;26(8):1619-26.

8. Prefeitura da Cidade do Rio de Janeiro (BR). Armazém de Dados: Instituto Pereira Passos [acesso em 2014 Jul 31]. Disponível em: http://portalgeo.rio.rj.gov.br

9. Castro CBA, Costa PA, Rufino-Netto A, Maciel ELN, Kritski AL. Avaliação de um escore clínico para rastreamento de suspeitos de tuberculose pulmonar. Rev Saúde Pública. 2011;45(6):1110-6.

10. Mota MRT. Prevalência de sintomáticos respiratórios nos consultantes de primeira vez no Município de Fortaleza. Bol Pneumol Sanit. 2004;12(2):85-90.

11. Rodrigues ILA, Cardoso NC. Detecção de sintomáticos respiratórios em serviços de saúde da rede pública de Belém, Pará, Brasil. Rev Pan-Amazônica Saúde. 2010;1(1):67-71.

12. Bastos LGV, Fonseca LS, Mello FCQ, Ruffino-Netto A, Golub JL, Conde MB. Prevalence of pulmonary tuberculosis among respiratory symptomatic subjects in an out-patient primary health unit. Int J Tuberc Lung Dis. 2007;11(2):156-60.

13. Marín MAV, Cholula CT, Castillo RO. Tuberculosis pulmonar entre sintomáticos respiratorios detectados en las unidades de salud de la SSA, en el estado de Tlaxcala, México. Rev Inst Nac Enfermedades Respir. 1999;12(1):29-34.

14. Santha T, Garg R, Subramani R, Chandrasekaran V, Selvakumar N, Sisodia RS, et al. Comparison of cough of 2 and 3 weeks to improve detection of smearpositive tuberculosis cases among out-patients in India. Int J Tuberc Lung Dis. 2005;9(1):61-8.

15. Gustafson P, Gomes VF, Vieira CS, Rabna P, Seng R, Johansson P, et al. Tuberculosis in Bissau: incidence and risk factors in an urban community in sub-Saharan Africa. Int J Epidemiol. 2004;33(1):163-72.

16. Alcântara CCS, Kritski AL, Ferreira VG, Façanha MC, Pontes RS, Mota RS, et al. Fatores associados à tuberculose pulmonar em pacientes que procuram serviços de saúde de referência para tuberculose. J Bras Pneumol. 2012;38(5):622-9.

17. den Boon S, van Lill SW, Borgdorff MW, Verver S, Bateman ED, Lombard CJ, et al. Association between smoking and tuberculosis infection: a population survey in a high tuberculosis incidence area. Thorax. 2005;60(7):555-7. 
18. Job JRPP, Prado PEBS, Vranjac S, Duarte PC. Comparação de dados epidemiológicos da tuberculose pulmonar em Sorocaba, SP, Brasil, em uma década (1986 - 1996). Rev Saúde Pública. 1998;32(6):596-7.

19. Vieira AA, Ribeiro SA, Siqueira AM, Galesi VMN, Santos LAR, Golub JE. Prevalence of patients with respiratory symptoms through active case finding and diagnosis of pulmonary tuberculosis among prisoners and related predictors in a jail in the city of Carapicuíba, Brazil. Rev Bras Epidemiol. 2010;13(4):641-50.

20. Monroe AA, Gonzales RIC, Palha PF, Sassaki CM, Ruffino-Netto A, Vendramini SHF, Villa TCS. Envolvimento de equipes da atenção básica à saúde no controle da tuberculose. Rev Esc Enferm USP. 2008;42(2):262-67.

21. Oliveira MF, Arcêncio RA, Ruffino-Netto A, Scatena LM, Palha PF, Villa TCS. The front door of the Ribeirão Preto health system for diagnosing tuberculosis Rev Esc Enferm USP 2011;45(4):898-904.

22. Loureiro RB, Villa TCS, Ruffino-Netto A, Peres RL, Braga JU, Zandonade E, et al. Acesso ao diagnóstico da tuberculose em serviços de saúde do município de Vitória, ES, Brasil. Ciênc Saúde Coletiva. 2014;19(4):1233-44.
23. Muniz JN, Palha PF, Monroe AA, Gonzales RC, Ruffino-Netto A, Villa TCS. A incorporação da busca ativa de sintomáticos respiratórios para o controle da tuberculose na prática do agente comunitário de saúde. Ciênc Saúde Coletiva. 2005;10(2):315-24.

24. Maciel ELN, Vieira RCA, Milani EC, Brasil M, Fregona G, Dietze R. O agente comunitário de saúde no controle da tuberculose: conhecimentos e percepções. Cad Saúde Pública. 2008; 24(6):1377-86.

25. Maciel ELN, Araújo WK, Giacomin SS, Jesus FA, Rodrigues PM, Dietze R. O conhecimento de enfermeiros e médicos que trabalham na Estratégia de Saúde da Família acerca da tuberculose no município de Vitória (ES): um estudo de corte transversal. Ciênc Saúde Coletiva. 2009;14(Suppl 1):1395-402.

\section{Endereço de correspondência}

Monalisa Garcia de Oliveira

Universidade Iguaçu

Rua Abílio Augusto Távora, 2134

Jardim Nova Era - Nova Iguaçu - Rio de Janeiro - RJ

CEP 26.275-580

E-mail: monalisagoliveira@gmail.com 